\title{
20 ans de recherche au LPES dans le cadre des LARD
}

\author{
P. IACCONI ${ }^{1}$, D. LAPRAZ ${ }^{1}$, M. BENABDESSELAM ${ }^{1}$, F. MADY ${ }^{1}$, F. WROBEL ${ }^{1}$, \\ R. BINDI ${ }^{1}$, S. HACHEM ${ }^{1}$
}

(Manuscrit reçu le 6 novembre 2006, accepté le 28 février 2007)

\begin{abstract}
RÉSUMÉ Le Laboratoire de Physique Électronique des Solides (LPES) de l'université de NiceSophia Antipolis (UNSA) est membre fondateur des « Laboratoires Associés en Dosimétrie et en Radiophysique » (LARD). Depuis, les travaux effectués au LPES s'inscrivent dans le cadre des LARD. Nous essayons, ici, de retracer vingt ans de recherches effectuées en relation avec la dosimétrie des rayonnements ionisants et l'interaction rayonnement-matière.

ABSTRACT The twenty-years-old LARD association at LPES.

In the mid-eighties, the Electronics and Solid-State Physics Laboratory (LPES) of the University of Nice Sophia-Antipolis was one of the founding members of the socalled "Associated Laboratories in Radiophysics and Dosimetry" (LARD). Since this period, the LPES research has always been part of the LARD. This article tries to recount the LPES activity related to the dosimetry of ionizing radiations over the last twenty years.

Keywords: dosimetry / TLD / ionising radiation / OSL / modelling
\end{abstract}

\section{Introduction}

Il y a une vingtaine d'années, l'équipe de «Luminescence » du Laboratoire d'Émission Électronique et de Luminescence (LEEL) de l'université de Nice, devenue en 1990 le Laboratoire de Physique Électronique des Solides (LPES), participait - sous l'impulsion de D. Blanc et G. Portal - à la fondation des «Laboratoires Associés en Dosimétrie et en Radiophysique » (LARD). Cette équipe était alors composée de P. Keller, P. Iacconi et D. Lapraz et travaillait sur les propriétés thermoluminescentes de l'alumine alpha. Par la suite, l'équipe s'est agrandie et ses thèmes de recherches ont évolué.

Nous nous proposons de retracer ici les différents résultats obtenus dans le cadre des LARD et de leur environnement. Ce dernier était important car il conférait au LPES le statut d'équipe d'accueil de la formation doctorale de Physique Médicale de Toulouse (UPS) et nous plaçait dans une relation contractuelle avec l'IPSN (encore CEA à l'époque).

${ }^{1}$ Université de Nice-Sophia Antipolis, Laboratoire de Physique Électronique des Solides (LPES), Centre de Recherche sur les Solides et leurs Applications (CRESA), EA 1174, Parc Valrose, 06108 Nice Cedex 2, France. 
Par la suite, les recherches décrites ont toujours été développées en amont de la dosimétrie des rayonnements ionisants, avec le soutien du CEA, de la DGA ou de l'IPSN ; elles ont donné lieu à une quinzaine de thèses et ont fait l'objet de nombreuses communications aux divers Congrès de Dosimétrie en général et aux journées des LARD en particulier.

On notera cependant que, pour le LPES, le véritable point de départ de cette rétrospective se situe en 1980, à la « 6th International Conference on Solid State Dosimetry » de Toulouse où se nouèrent les premiers contacts avec le monde de la dosimétrie des rayonnements ionisants.

Des thèmes de recherches effectuées au LPES dans le cadre de la dosimétrie des rayonnements ionisants, on peut extraire six grands domaines d'application s'appuyant sur la recherche fondamentale. Nous ne reporterons ici que des résultats en relation avec la dosimétrie et la radiophysique :

1. la caractérisation et la modélisation des phénomènes dosimétriques de l'alumine ;

2. le coton aluminé (CEA, DGA, sources de contrats) ;

3. la dosimétrie haute température $\left(\mathrm{CaSO}_{4}\right.$ et dérivés);

4. la dosimétrie en milieu hostile (diamant et autres semiconducteurs à large gap) ;

5. l'interaction nucléon-matière ;

6. la radiothérapie du cancer.

\section{Les propriétés dosimétriques de l'alumine}

\subsection{Phototransfert (1980-1990)}

La thermoluminescence (TL) est le phénomène de luminescence que l'on observe lorsqu'un isolant (ou semiconducteur) est chauffé après avoir subi une irradiation par un rayonnement ionisant. L'interprétation du phénomène est liée au fait que les imperfections du matériau «piègent » les porteurs de charge libérés lors de l'irradiation et que ces charges ne peuvent être dépiégées que par un apport d'énergie thermique adéquat. Habituellement, on élève la température du matériau avec une vitesse de chauffage constante jusqu'à une température suffisamment élevée de façon à vider tous les pièges. On obtient ainsi une succession de pics de TL aparaîssant dans l'ordre croissant des températures et correspondant aux énergies d'activation des pièges concernés.

Si le dépiégeage d'un électron (ou d'un trou) est suivi par le passage par la bande de conduction (ou de valence), on peut observer un phénomène de conductivité électrique thermostimulée (CETS). Si l'électron dépiégé acquiert assez s'énergie pour franchir la barrière de potentiel de surface, il peut sortir dans le vide, donnant ainsi naissance à l'émission exoélectronique thermostimulée (EETS). 
La dosimétrie par thermoluminescence (TLD) utilise la relation existant entre l'intensité des pics de TL et la dose de rayonnement ionisant déposée dans le matériau. La température d'irradiation est la température ambiante et le pic dosimétrique de TL se trouve autour de $250{ }^{\circ} \mathrm{C}$ mais les pièges profonds nécessitent souvent une température de $500{ }^{\circ} \mathrm{C}$ pour être vidés.

Le phénomène de phototransfert de thermoluminescence (PTTL) intervient lorsque la température atteinte lors du chauffage n'est pas assez élevée et que les pièges profonds ne sont pas entièrement vidés. L'exposition à la lumière visible ou UV (ce qui est le cas de l'éclairage du laboratoire) peut alors provoquer une redistribution des populations des pièges entraînant notamment un remplissage partiel des pièges peu profonds (et dosimétriques). Ce phénomène de PTTL peut également intervenir sur un matériau non chauffé et fausser ainsi la lecture de la dose emmagasinée. En revanche, si on connaît bien le comportement du matériau, on peut utiliser le PTTL pour effectuer une deuxième lecture de dose.

Les travaux consacrés à ce sujet concernaient le phototranfert présenté par les phénomènes de TL (Lapraz et al., 1988 ; Sayadi, 1988 ; Iacconi et al., 1986 ; Lapraz et Iacconi, 1985), l'EETS (Iacconi et al., 1997, 1990) et la CETS (Lapraz et al., 1993).

\subsection{Les phénomènes thermostimulés (1990-2000)}

Une des activités importantes du LPES concerne l'étude des phénomènes thermostimulés (PTS) du point de vue fondamental. L'alumine alpha s'avère être un support idéal pour la validation des modélisations subséquentes. Un certain nombre de thèses et la construction de dispositifs appropriés ont permis de connaître les réponses d'EETS (Addi, 1990) et de CETS (Boutayeb, 1993). Mais, très vite, il apparût qu'il fallait pouvoir détecter simultanément les PTS pour pouvoir les comparer de façon précise (Iacconi et al., 1993), bien que cela soulève une impossibilité technique. En effet, la TL et la CETS sont des phénomènes de volume tandis que l'EETS est un phénomène de surface. Par ailleurs, la TL peut être mesurée sur un matériau mono ou polycristallin, alors que la CETS doit être obtenue sur un monocristal et que l'EETS se mesure mieux sur une poudre. Enfin, la présence d'électrodes nécessaires à la mesure de la CETS gêne la détection de la TL et de l'EETS. La conséquence est que nous avons construit deux appareillages : (i) le premier mesure la TL et l'EETS (Petel et al., 1996 ; Petel, 1994), (ii) le second détecte la TL et la CETS. La TL joue ainsi le rôle de dénominateur commun. Cette manière de procéder est indispensable pour s'affranchir des gradients thermiques - différents - introduits par les dispositifs de chauffage.

Par la suite, les travaux de Molnar (2000) et de Vinceller (2002) précisèrent le rôle joué par le phénomène d'extinction thermique de la luminescence sur la 
mesure de la TL et caractérisèrent les mécanismes de TL du pic dosimétrique de l'alumine alpha (Molnar et al., 2002, 1999).

\subsection{Dopage de l'alumine (1995-2000; CRESA)}

Dans les années 1990, le LPES s'associa au département de Chimie Physique des Processus Industriels (CPPI) de l'École des Mines de St. Étienne pour former le Centre de Recherche sur les Solides et leurs Applications (CRESA). Le CPPI (maintenant Sciences des Processus Industriels et Naturels, SPIN) fut représenté aux LARD par Guilhot et contribua à de nombreux travaux sur les propriétés dosimétriques de l'alumine alpha par le biais de thèses co-dirigées par le LPES et Guilhot puis Grosseau de SPIN. Les thèmes de recherche se basent sur le fait que la TL de l'alumine alpha est fortement tributaire de la stoechiométrie en oxygène (Papin et al., 1999). Il est, en effet, bien connu que les propriétés de TL de l'alumine sont liées à la présence de lacunes d'oxygène susceptibles de piéger un ou deux électrons (centres $\mathrm{F}^{+}$ou F). La compréhension des mécanismes de TL passe donc par la maîtrise de ces lacunes. Deux techniques sont disponibles pour cela : (i) le traitement thermique en milieu réducteur, qui favorise la présence de lacunes d'oxygène (Molnar et al., 2000 ; Papin et al., 1996) et (ii) le dopage par des éléments divalents $\mathrm{M}^{2+}$ se substituant à $\mathrm{Al}^{3+}$ qui favorise la présence de lacunes d'oxygène par compensation de charge (Salle, 2003 ; Ferey, 2002 ; Ferey et al., 2002, 2001). A contrario, un traitement thermique oxydant ou un dopage par des éléments tétravalents a plutôt tendance à supprimer les lacunes d'oxygène et à créer des lacunes d'aluminium (Papin, 1997 ; Papin et al., 1997). L'étude du dopage par le $\mathrm{Cr}^{3+}$ contribue également à la compréhension des phénomènes de TL (Lapraz et al., 1991).

\subsection{La modélisation (depuis 1995)}

La modélisation des PTS a pour but de caractériser les pièges associés aux pics dosimétriques en confrontant les courbes calculées aux thermogrammes expérimentaux. Elle doit en principe rendre compte de la TL, de la CETS et de l'EETS simultanément.

En pratique, la très grande majorité des modèles développés dans la littérature s'appuient sur un ensemble d'équations différentielles non linéaires couplées rendant compte des cinétiques de piégeage, de dépiégeage, de recombinaison et d'exoémission en fonction de la température. La résolution de ce jeu d'équations s'est d'abord effectuée de manière analytique, au prix d'approximations importantes limitant la validité des modèles et donc leur intérêt. On a ainsi établi 
les modèles cinétiques dits de «premier ordre », de «second ordre », ou encore "d'ordre généralisé », ne considérant qu'un seul piège actif, utilisant l'approximation dite de «quasi-équilibre » et négligeant l'EETS. L'amélioration incessante des moyens de calcul informatiques a, par la suite, ouvert la voie aux «simulations » des PTS basées sur la résolution numérique des équations. Cela a permis de s'affranchir des approximations les plus restrictives et d'envisager des modèles plus réalistes, et donc plus complexes, incluant par exemple plusieurs pièges actifs et l'EETS.

La figure 1 donne un exemple de modélisation de la TL de l'alumine $\alpha$ par un modèle à 4 pièges ( 3 actifs et un profond).

La modélisation des PTS au LPES s'est aussi articulée autour d'aspects analytiques et numériques. Son originalité a été de mettre en évidence les relations entre les PTS détectés simultanément et d'étudier le rôle de l'EETS, souvent négligée à tort dans d'autres travaux.

Sur le plan analytique, une technique d'extraction des profondeurs de pièges s'appuyant sur la détection simultanée de la TL et de la CETS a été proposée à partir d'un modèle reprenant les approximations de premier et second ordre (Bindi et al., 1994). Les travaux réalisés sur l'EETS de l'alumine alpha ont par ailleurs montré que l'exoémission de ce matériau (pics B' et D') était correctement décrite par un processus thermoïonique (Guissi, 1998 ; Guissi et al., 1998 ; Bindi et al., 1997). Suivant cette hypothèse, les mesures simultanées de TL et d'EETS ont conduit à une estimation de l'affinité électronique de ces pics (Bindi et al., 1997). Notons enfin que la généralisation d'un formalisme existant (modèle de Levandowski), corrigé de façon à y inclure l'EETS, nous a permis d'expliquer le décalage observé entre les pics de PTS associés à un même piège (Guissi et al., 1999).

Les raffinements apportés par les modèles de simulation numérique ont principalement consisté à tenir compte du gradient thermique existant entre la face chauffée de l'échantillon et la face de lecture (modèle dit «multicouche »), puis à considérer plusieurs pièges actifs afin de mieux modéliser les matériaux réels (modèles dits « interactifs » (Vinceller, 2002 ; Vinceller et al., 2002)). Le modèle multicouche a fourni une estimation corrigée de l'affinité effective et de la profondeur des pièges associés aux pics B'et D' (Berkane-Krachai et al., 2002 ; Berkane-Krachai, 2001). Les modèles interactifs ont quant à eux montré comment la présence de plusieurs pièges modifiait la réponse des PTS. Ils sont désormais utilisés pour étudier l'évolution de cette réponse en fonction de la dose absorbée et en tirer des conclusions pratiques quant à l'interprétation des données expérimentales (Mady et al., 2006 ; Mady et al., 2005). 


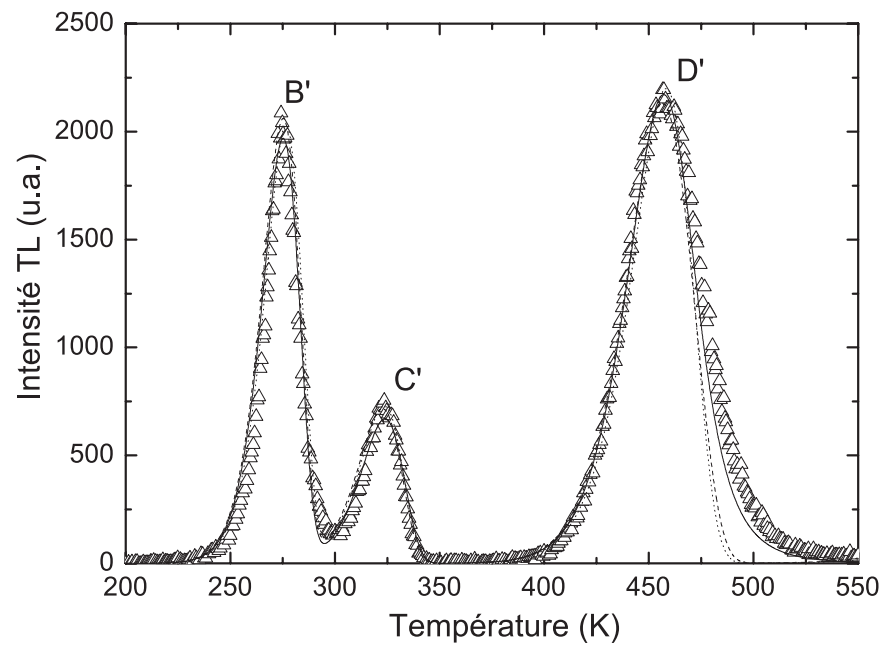

Figure 1 - Exemple de modélisation des pics $\mathrm{TL}$ de l'alumine alpha $\left(\mathrm{Al}_{2} \mathrm{O}_{3}: \mathrm{C}\right)$ à l'aide d'un modèle numérique à 4 pièges dont 3 actifs (associés aux pics B', $C^{\prime}$ et $D^{\prime}$ ) et un profond. Symboles ouverts $(\Delta)$ : mesure pour vitesse de chauffe à $0,5 \mathrm{~K} / \mathrm{s}$; ligne en trait plein $(-)$ : modèle considérant une section efficace de piégeage très inférieure à la section efficace de recombinaison avec les densités et profondeurs de pièges suivantes: $3,8 \times 10^{11} \mathrm{~cm}^{-3}$ et $0,731 \mathrm{eV}$ pour $\mathrm{B}^{\prime}, 1,3 \times 10^{11} \mathrm{~cm}^{-3}$ et $0,866 \mathrm{eV}$ pour $C^{\prime}, 4,9 \times 10^{11} \mathrm{~cm}^{-3}$ et 1,229 $\mathrm{eV}$ pour $\mathrm{D}^{\prime}$, densité de pièges profonds négligeable; ligne tirets (- - - : : modèle considérant une section efficace de piégeage très inférieure à la section efficace de recombinaison avec les densités et profondeurs de pièges suivantes : $3,0 \times 10^{12} \mathrm{~cm}^{-3}$ et $0,729 \mathrm{eV}$ pour $\mathrm{B}^{\prime}, 9,5 \times 10^{11} \mathrm{~cm}^{-3}$ et $0,862 \mathrm{eV}$ pour $C^{\prime}, 3,2 \times 10^{12} \mathrm{~cm}^{-3}$ et 1,226 eV pour $D^{\prime}$, densité de pièges profonds de $2,85 \times 10^{12} \mathrm{~cm}^{-3}$; ligne pointillés $(\cdots)$ : modèle considérant une section efficace de piégeage égale à celle de recombinaison avec les densités et profondeurs de pièges suivantes : $4,0 \times 10^{12} \mathrm{~cm}^{-3}$ et $0,725 \mathrm{eV}$ pour $\mathrm{B}^{\prime}, 8,5 \times 10^{11} \mathrm{~cm}^{-3}$ et 0,862 $\mathrm{eV}$ pour $C^{\prime}, 1,95 \times 10^{12} \mathrm{~cm}^{-3} \mathrm{et} \mathrm{1,222} \mathrm{eV}$ pour $D^{\prime}$, densité de pièges profonds de $3,2 \times 10^{12} \mathrm{~cm}^{-3}$. On remarque que des hypothèses physiques très distinctes à l'échelle des pièges peuvent conduire à des pics TL non discernables à l'échelle expérimentale. On illustre ainsi une limitation importante de la technique concernant sa capacité à caractériser les pièges.

Modelling of the TSL peaks of alpha alumina $\left(\mathrm{Al}_{2} \mathrm{O}_{3}: \mathrm{C}\right)$. Examples of fits obtained from a 4 traps models (3 active traps associated with peaks $B$ ', $C^{\prime}$ et $D$ ' and one deep trap). Open symbol $(\Delta)$ : measurement at $0.5 \mathrm{~K} \mathrm{~s}^{-1}$; solid line (-): model using a trapping cross section much smaller than that of recombination and the following trap densities and depths: $3.8 \times 10^{11} \mathrm{~cm}$ and $0.731 \mathrm{eV}$ for $\mathrm{B}^{\prime}, 1.3 \times 10^{11} \mathrm{~cm}^{-3}$ and $0.866 \mathrm{eV}$ for $\mathrm{C}^{\prime}, 4.9 \times 10^{11} \mathrm{~cm}^{-3}$ and $1.229 \mathrm{eV}$ for $\mathrm{D}^{\prime}$, negligible density of deep traps; dashed line (- . - -): model using a trapping cross section much smaller than that of recombination and the following trap densities and depths: $3.0 \times 10^{12} \mathrm{~cm}$ and $0.729 \mathrm{eV}$ for $\mathrm{B}^{\prime}, 9.5 \times 10^{11} \mathrm{~cm}^{-3}$ and $0.862 \mathrm{eV}$ for $C^{\prime}, 3.2 \times 10^{12} \mathrm{~cm}^{-3}$ and $1.226 \mathrm{eV}$ for $D^{\prime}$, deep traps: $2.85 \times 10^{12} \mathrm{~cm}^{-3}$; dotted line $(\cdots)$ : model using a trapping cross section equal to that of recombination and the following trap densities and depths: $4.0 \times 10^{12} \mathrm{~cm}^{-3}$ and $0.725 \mathrm{eV}$ for $\mathrm{B}^{\prime}, 8.5 \times 10^{11} \mathrm{~cm}^{-3}$ and $0.862 \mathrm{eV}$ for $\mathrm{C}^{\prime}, 1.95 \times 10^{12} \mathrm{~cm}^{-3}$ and $1.222 \mathrm{eV}$ for $\mathrm{D}^{\prime}$, deep traps: $3.2 \times 10^{12} \mathrm{~cm}^{-3}$. One has to notice that quite different physical hypotheses at the trap scale can yield very similar TSL peaks. This illustrates a basic limitation of the technique, i.e. of the attempt to investigate trap parameters by fitting TSL curves. 


\section{Le coton aluminé}

\subsection{Le coton aluminé (1982-1992; CEA)}

L'idée du coton aluminé a germé au cours d'un travail contractuel que le LPES effectuait pour le compte du SIDR (devenu au fil des restructurations IPSN/DPHD/Service de dosimétrie) avec Portal, Barthe et Petel. Ce travail s'intitulait : « Développement d'une technique individuelle de substitution en cas d'irradiation accidentelle » et avait pour objet d'établir une méthode permettant de mesurer, par des méthodes thermostimulées, la dose accidentelle absorbée par des objets portés par l'individu accidenté (vêtements, cheveux, dents, etc.).

Les recherches portant sur l'exoémission du coton n'ayant rien donné (Barthe et al., 1984, 1983), nous décidâmes de fabriquer un tissu destiné à l'habillement de travailleurs soumis à un risque d'irradiation. Nous prîmes contact avec le Pr Freytag et Huet du Centre de Recherches Textiles de Mulhouse. Après avoir retenu l'idée d'un tissu de coton recouvert d'une couche d'alumine, nous dressâmes le cahier des charges et nous nous lançâmes dans les essais préliminaires. Les critères de choix de l'alumine englobaient la granulométrie (éviter les tissus qui râpent) et la réponse de TL (seuil de détection). Ils nous permirent de sélectionner une alumine industrielle oxydée (Fig. 2) moins sensible à la lumière ambiante que l'alumine réduite et de granulométrie convenable. Les essais suivants portèrent sur des éprouvettes de coton, préparées par trois méthodes différentes et ayant reçu diverses charges d'alumine : (i) réponse de TL en absence d'oxygène (Fig. 3), (ii) tests de portabilité (rigidité, allergie, effet de la lumière ambiante ou solaire, etc.), (iii) tests de lavabilité (nettoyage à sec, lavage à chaud, nombre de lavage, etc.) (AlessandriFraccaro, 1989). Les résultats furent présentés à la « IXth International Conference on Solid State Dosimetry » de Vienne en novembre 1989 (Lapraz et al., 1990).

Après le départ de Portal, les travaux sur le coton aluminé furent suspendus. Le planning prévoyait alors de fabriquer trois blouses avec le tissu retenu et de les faire porter à des travailleurs dans des conditions normales suivant un cycle de porter-lavage défini. Le but était de montrer la faisabilité et le confort d'une telle blouse. L'étape finale aurait été l'irradiation d'un mannequin anthropomorphe revêtu d'une telle blouse et la reconstitution de la cartographie de la dose absorbée par le mannequin.

Après avoir envisagé de faire breveter cette invention, l'IPSN/DPHD laissa l'exploitation du coton chargé en alumine à la DGA.

\subsection{Le coton aluminé (1993-1998; DGA)}

En 1993, nous entamâmes une collaboration avec Dhermain, Laugier et Serbat de la DGA/DRET/ETCA, Centre du Bouchet - Arcueil Cedex, sur le thème 


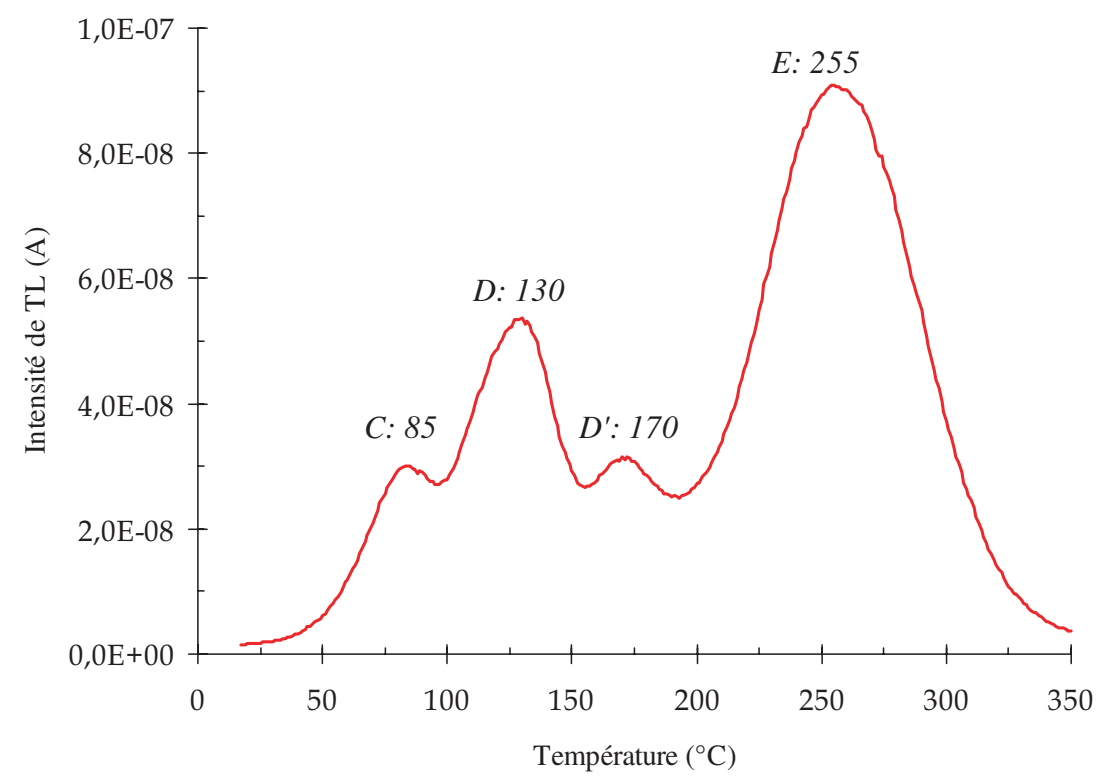

Figure 2 - Courbe de TL obtenue après irradiation X (kerma dans l'air de 3 Gy) à température ambiante sur une alumine réduite.

TL curve after X-rays irradiation (kerma in air of $3 \mathrm{~Gy}$ ) at room temperature on a reduced alumina.

«Collaboration dans la recherche d'une méthode de dosimétrie en cas d'accident en champ mixte $\gamma, \mathrm{n} »$.

Il s'agissait d'utiliser le coton aluminé comme un détecteur souple pouvant épouser des formes particulières de façon à valider des codes de calcul (Benabdesselam et al., 1998, 1997, 1996a, 1996b).

\section{Dosimétrie haute température (HT) et imagerie OSL (depuis 1993)}

Une des activités de recherche développées au LPES concerne la caractérisation des propriétés thermoluminescentes de $\mathrm{CaSO}_{4}$ dopé ou codopé par des éléments de terres rares.

La plupart des travaux réalisés ont été effectués en collaboration avec le CEM2 de l'université de Montpellier II (Gasiot, Fesquet, Prevost et Dusseau) et avec Lakshmanan du Centre Indira Gandhi pour la recherche atomique de Kalpakkam (Inde). Les comportements suivants ont été particulièrement étudiés : (i) influence 


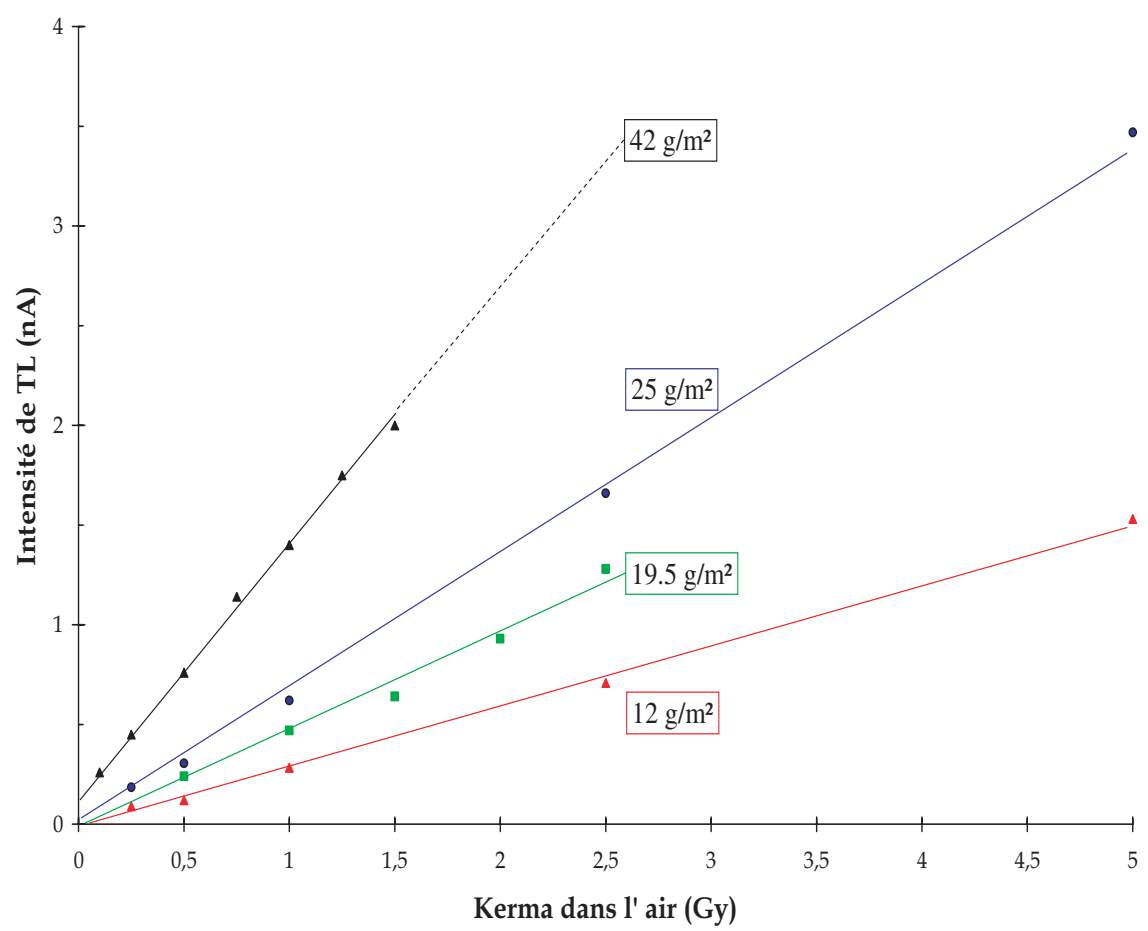

Figure 3 - Courbes d'étalonnage. Chaque courbe correspond aux variations de l'intensité de TL du pic dosimétrique en fonction du kerma dans l'air suite à des irradiations y $\left({ }^{60} \mathrm{Co}\right)$ pour 4 teneurs en alumine dans le coton.

Calibration curves. Each curve corresponds to TL intensity variations versus kerma in air after $y$ irradiation $\left({ }^{60} \mathrm{Co}\right)$ for 4 alumina contents in cotton.

des méthodes de préparation sur la réponse de TL (Lakshmanan et al., 2005 ; Gerôme et al., 1996), (ii) application à la dosimétrie HT (Fig. 4) telle qu'elle se trouve dans les centrales nucléaires $\left(100-300{ }^{\circ} \mathrm{C}\right)$ ou au voisinage des déchets radioactifs (Goyet et al., 1996, 1993 ; Goyet, 1993), (iii) mise en évidence d'une émission de réseau et de processus de transferts d'énergie entre le réseau et les dopants de terres rares (Gerôme, 1997 ; Lapraz et al., 1996), (iv) rôle du dopage par l'europium qui peut se trouver sous deux états de valence avec des proportions $\mathrm{Eu}^{2+} / \mathrm{Eu}^{3+}$ qui varient énormément suivant les conditions de préparation ou d'irradiation (Lapraz et al., 2004, 2000).

Ces dernières années, nous nous sommes intéressés aux applications dosimétriques par TL, par photoluminescence et par OSL (Optical Stimulated Luminescence) de $\mathrm{CaSO}_{4}: \mathrm{Ce}$ (Lapraz et al., 2002) et $\mathrm{CaSO}_{4}: \mathrm{Sm}$ pour la 


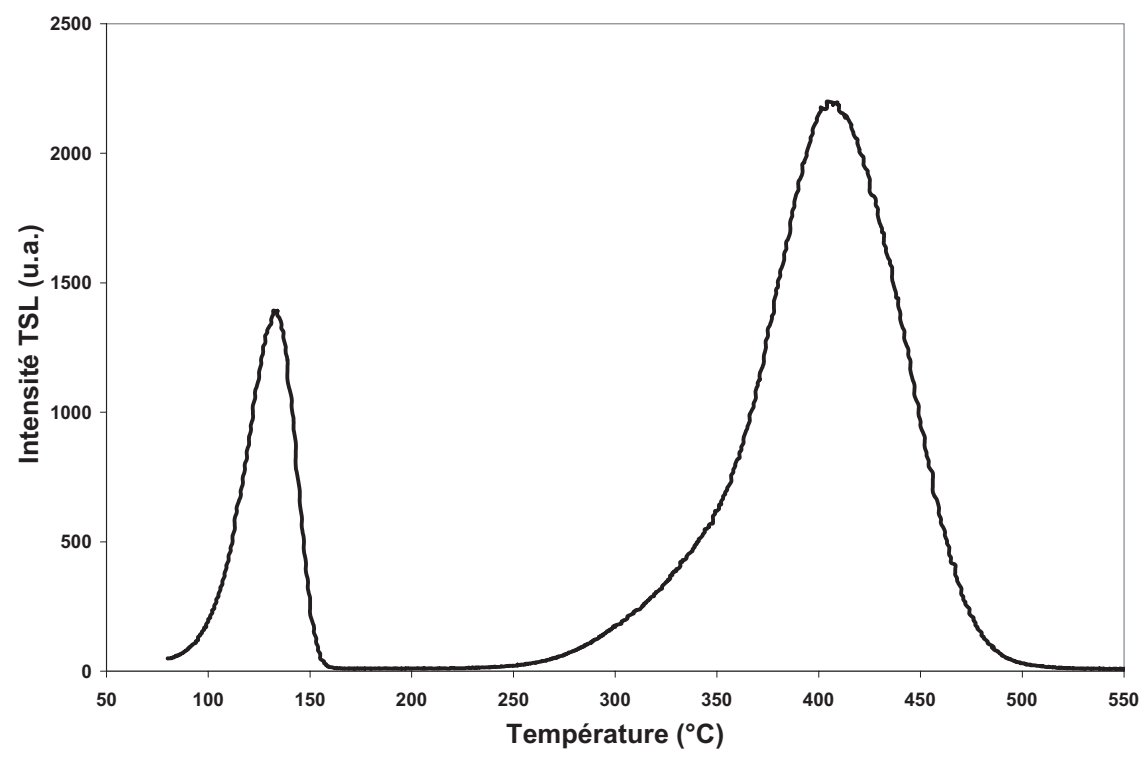

Figure 4 - Courbe de thermoluminescence de $\mathrm{CaSO}_{4}: 0,1 \%$ Ce après irradiation $\mathrm{X}(45 \mathrm{kV})$ de $100 \mathrm{~Gy}$ à $20{ }^{\circ} \mathrm{C}$ (vitesse de chauffage : $1{ }^{\circ} \mathrm{C} / \mathrm{s}$ ).

TSL curve of $\mathrm{CaSO}_{4}: 0.1 \%$ Ce after X-irradiation (45 kV-100 Gy) at $20{ }^{\circ} \mathrm{C}$ (heating rate of $\left.I^{\circ} \mathrm{C} / \mathrm{s}\right)$.

dosimétrie haute température et de $\mathrm{SrS}: \mathrm{Ce}, \mathrm{Sm}$ (Fig. 5) et $\mathrm{CaS}: \mathrm{Ce}, \mathrm{Sm}$ pour l'imagerie OSL.

\section{Le diamant (depuis 2000)}

Soucieux de valider les modèles mis au point au LPES et jusqu'ici testés uniquement sur l'alumine alpha, nous nous sommes mis en quête de matériaux ayant une faible (voire négative) affinité électronique effective. Très vite le diamant CVD (Chemical Vapour Deposition) s'avéra être un très bon candidat doté, de plus, de propriétés dosimétriques intéressantes : (i) équivalence tissu, (ii) non-toxicité, (iii) résistance aux agents désinfectants, (iv) bonne réponse aux rayonnements, etc. Il peut être utilisé comme détecteur actif (en mode chambre d'ionisation) ou passif (en thermoluminescence). C'est pour ces raisons que nous avons recentré nos activités sur l'étude des propriétés dosimétriques par thermoluminescence du diamant CVD. Les couches minces de diamant sont élaborées au NRL (Butler, Naval Research Laboratory, Washington DC, USA). 


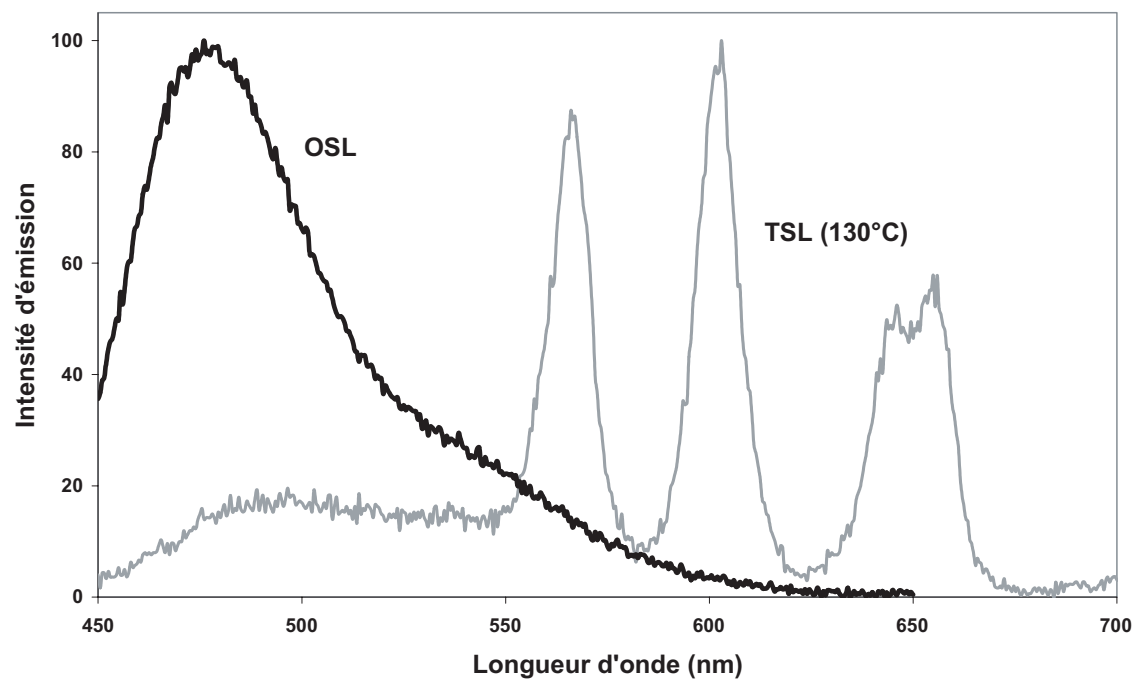

Figure 5 - Spectres d'émission de SrS:Ce,Sm après excitation UV $(254 \mathrm{~nm})$ à $20^{\circ} \mathrm{C}$ en $\mathrm{TSL}$ (pic à $130^{\circ} \mathrm{C}$ ) et en $\mathrm{OSL}\left(20^{\circ} \mathrm{C}\right)$.

TSL (peak at $\left.130^{\circ} \mathrm{C}\right)$ and $\mathrm{OSL}\left(20^{\circ} \mathrm{C}\right)$ spectra of SrS:Ce,Sm after $\mathrm{UV}(254 \mathrm{~nm})$-excitation at $20{ }^{\circ} \mathrm{C}$.

Les premiers films de diamant CVD, provenant d'un même wafer et irradiés par photons (X ou $\gamma$ ), se sont avérés inaptes à l'élaboration de dosimètres de routine. La disparité des sensibilités observées a été attribuée à l'instabilité des conditions de synthèse du wafer (Benabdesselam et al., 2000). Par la suite, ces conditions ont été améliorées et nous avons pu disposer de substrats de diamant polycristallin, fabriqués à faible coût et à grande échelle, ayant des propriétés dosimétriques acceptables (Benabdesselam et al., 2003).

Les films de diamant CVD peuvent également être utilisés pour la dosimétrie des rayons UV. Dans ce cas, ils conviennent très bien pour les UV-C (soudure à l'arc) mais sont inappropriés dans la gamme 280-400 nm, c'est-à-dire pour la dosimétrie UV-A ou -B (Benabdesselam et al., 2001).

Par ailleurs, l'effet néfaste de l'exposition à la lumière du laboratoire d'un dosimètre diamant CVD irradié a été mis en évidence et caractérisé. Il se traduit par une perte de l'information dosimétrique c'est-à-dire un affaiblissement de l'intensité du pic dosimétrique de TL (fading optique). Des travaux en cours ont pour but de quantifier les effets du très net phénomène d'extinction thermique de luminescence récemment identifiée sur les diamants CVD et font l'objet de la thèse de Petitfils. 


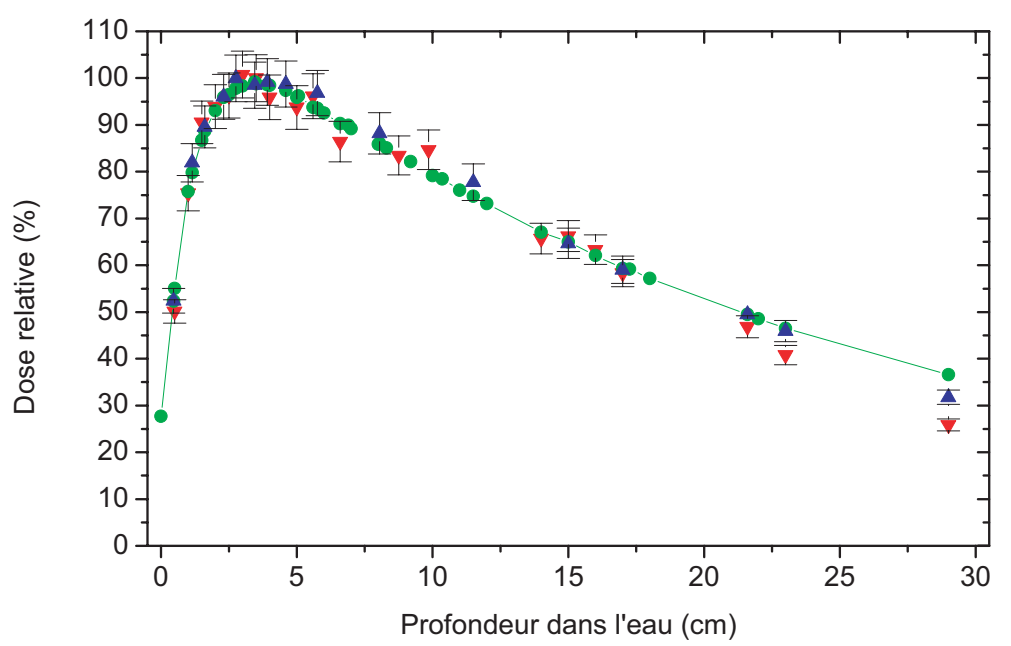

Figure 6-Comparaison entre les réponses d'une chambre d'ionisation et d'un dosimètre thermoluminescent à base de diamant CVD en termes de distribution de la dose relative dans l'eau pour un faisceau de photons de tension nominale $25 \mathrm{MV}$ délivré par l'accélérateur linéaire PRIMUS. Le champ est de $1 \times 10 \mathrm{~cm}^{2}$; la distance source échantillon (SSD) de $100 \mathrm{~cm}$ correspond à 9,7 mGy/MU (Unité Moniteur) sous une profondeur de $5 \mathrm{~cm}$ dans l'eau ; (a) : avec différents films CVD superposés dans l'eau le long du faisceau $(\nabla),(b)$ : avec un seul film de diamant placé à différentes profondeurs $(\mathbf{\Delta})$.

Comparison between CVD diamond TL dosemeter and ionisation chamber responses in terms of the relative dose distribution in water for the nominal voltage of $25 \mathrm{MV}$ photon beam from the PRIMUS linear accelerator. The field size is $10 \times 10 \mathrm{~cm}^{2}$ at an $S S D$ of $100 \mathrm{~cm}$ corresponding at $5 \mathrm{~cm}$ depth in water to $9.7 \mathrm{mGy} / \mathrm{MU}$; (a): with different CVD films superimposed all along the photon beam $(\nabla),(b)$ : with only one $C V D$ film placed at different depths $(\mathbf{\Delta})$.

Enfin, nous avons testé les possibilités présentées par les dosimètres de diamant CVD pour la radiothérapie (Fig. 6). Pour cela, nous avons comparé les performances dosimétriques (rendements en profondeur et de profil) du diamant CVD en mode TL et d'une chambre d'ionisation sous un faisceau d'irradiation photons de 25 MV. Les résultats obtenus (Petitfils et al., 2005 ; Benabdesselam et al., 2004) montrent l'aptitude de ce matériau pour une utilisation en radiothérapie.

\section{Interaction nucléon-matière (depuis 2003)}

La thématique « interaction des nucléons avec la matière » est abordée au LPES depuis 2003. Deux applications principales sont visées : le calcul de dose et les effets des neutrons avec les composants électroniques. Dans les deux cas, un effort tout particulier est mis sur l'aspect simulation avec le développement d'un code de 


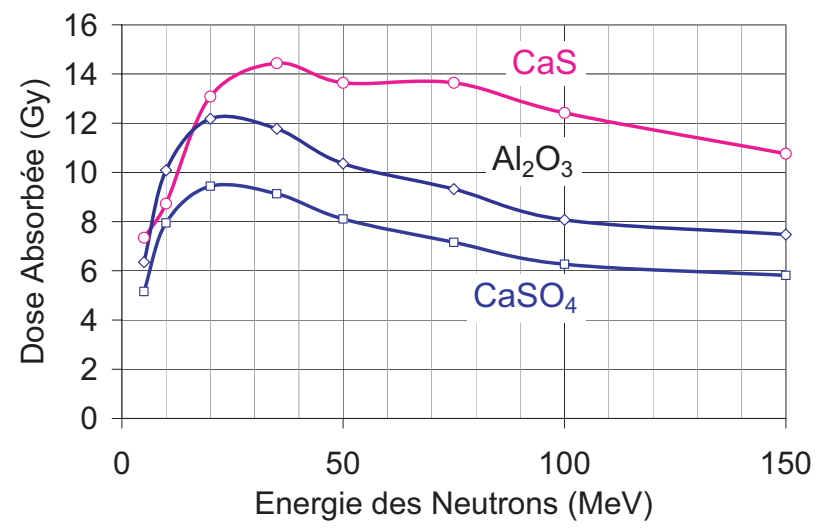

Figure 7 - Dose absorbée calculée avec MC-RED. La fluence est $10^{10}$ neutrons/cm ${ }^{2}$.

Total absorbed dose calculated with MC-RED. The fluence is $10^{10}$ neutrons $/ \mathrm{cm}^{2}$.

calcul dédié à l'interaction nucléon-matière. Le code ainsi développé se nomme MC-RED (Monte-Carlo Recoil Energy Determination) et permet de simuler, par une approche Monte Carlo, les réactions nucléaires induites dans la matière par des nucléons d'énergies comprises entre $100 \mathrm{keV}$ et $200 \mathrm{MeV}$. La particularité du code est de pouvoir fournir les énergies des différentes particules secondaires et, en particulier, celle du noyau de recul qui est capable de libérer localement toute son énergie cinétique (son pouvoir d'arrêt est important). Les réactions élastiques sont traitées par un appel direct au code bien connu ECIS (Équations Couplées par Itérations Séquentielles). Les réactions non-élastiques sont, quant à elles, traitées par les modèles de l'exciton et de l'évaporation de Hauser-Feschbach.

Une application directe du code est le calcul de dose dans différents matériaux (Fig. 7) et en particulier dans $\mathrm{Al}_{2} \mathrm{O}_{3}, \mathrm{CaS}$ et $\mathrm{CaSO}_{4}$ (Wrobel et al., 2004b). Puisque le code fournit les énergies de toutes les particules secondaires issues des réactions nucléaires, il est possible de calculer l'énergie déposée dans le matériau.

MC-RED est également utilisé pour l'évaluation de la fiabilité des composants électroniques en environnement naturel radiatif (neutrons atmosphériques mais aussi protons dans l'espace). Le couplage des données de physique nucléaire issue de MC-RED avec un simulateur de composants électroniques permet de prévoir l'effet électrique du passage d'un nucléon dans un tel composant et son éventuel dysfonctionnement. Un intérêt tout particulier est porté aux aléas logiques pouvant survenir dans les mémoires SRAM (changement accidentel de l'état d'un bit suite au passage d'une particule) (Wrobel et al., 2005, 2004a, 2003). 


\section{Radiothérapie du cancer (depuis 1997)}

Depuis son arrivée au LPES en 1997, Hachem y développe une nouvelle thématique axée sur l'application de la physique nucléaire à la médecine.

Ces travaux se font en collaboration avec le Centre A. Lacassagne de Nice et le CEA et se subdivisent en deux thèmes :

- la radiothérapie du cancer par capture de neutrons (Bourhis, 2000 ; BourhisMartin et al., 2002) ;

- la radiothérapie de conformation par modulation d'intensité (Serrano, 2006 ; Franchisseur et al., 2004 ; Marcié et al., 2003 ; Martin et al., 2003 ; Martin, 2002).

\section{Conclusion}

À la lueur de cette rétrospective, on se rend compte combien les « Laboratoires Associés en Dosimétrie et en Radiophysique » (LARD) ont stimulé les recherches effectuées au LPES. Durant la période allant jusqu'en 1992, le SDOS (CEA/IPSN) dirigé par Portal et conseillé par le Professeur Daniel Blanc soutint et finança les recherches fondamentales susceptibles d'être appliquées en dosimétrie des rayonnements ionisants. Cette période déboucha sur la définition d'un « tissu dosimétrique » destiné à habiller les travailleurs soumis à un risque d'irradiation. Le service de Portal jouait alors un rôle attracteur pour les cinq laboratoires fondateurs des LARD. Les travaux de qualification du coton aluminé reprirent sous l'égide de la DGA/ETCA et continuèrent jusqu'en 1998, date à laquelle la DGA abandonna le financement des recherches sur le vêtement dosimétrique. Une belle aventure prenait fin.

Entre temps, les LARD continuaient de se réunir annuellement (en général, en octobre), poursuivant ainsi le mouvement lancé par les membres fondateurs. Les relations n'étaient plus contractuelles mais étaient devenues amicales.

\section{RÉFÉRENCES}

Addi D. (1990) Réalisation d'un dispositif destiné à l'étude de l'émission exoélectronique entre 77 et 675 K. Caractérisation d'un phénomène de phototransfert dans l'alumine alpha thermostimulée de l'alumine à partir de $77 \mathrm{~K}$, Thèse de l'université Paul Sabatier-Toulouse.

Alessandri-Fraccaro M.F. (1989) Mise en évidence et étude d'un phototransfert de l'EETS de l'alumine alpha. Caractérisation des propriétés exoémissives et thermoluminescentes d'un tissu aluminé destiné à la dosimétrie des rayonnements ionisants en cas d'accident, Thèse de l'université Paul Sabatier-Toulouse.

Barthe J., Iacconi P., Lapraz D., Petel M., Portal G., Keller P. (1983) Electron emission from cotton fibres, Radiat. Prot. Dosim. 4, 151-153. 
Barthe J., Iacconi P., Lapraz D., Petel M., Keller P., Portal G. (1984) Use of cotton in accident dosimetry, Radiat. Prot. Dosim. 6, 125-128.

Benabdesselam M., Iacconi P., Lapraz D., Serbat A., Laugier J., Dhermain J. (1996a) Qualification of specially prepared clothing material for gamma dosimetry. Application to mixed neutronphoton radiation fields, Radiat. Prot. Dosim. 66, 125-128.

Benabdesselam M., Iacconi P., Lapraz D. (1996b) Cartographie de dose par tissu aluminé : état des recherches actuelles. Dans : $13^{\text {es }}$ Journées des LARD, 25 octobre 1996, Grenoble.

Benabdesselam M., Iacconi P., Lapraz D., Serbat A., Dhermain J., Laugier J. (1997) Characterization of a clothing material meant to gamma dosimetry in mixed neutron-gamma fields, Radiat. Prot. Dosim. 70, 461-465.

Benabdesselam M., Iacconi P., Lapraz D., Serbat A., Dhermain J. (1998) Cartography of absorbed doses by dosimetric textile. Testing in $(\mathrm{n}, \gamma)$ or $(\beta, \gamma)$ mixed field, Radioprotection 33, 63-71.

Benabdesselam M., lacconi P., Briand D., Butler J.E. (2000) Performance of CVD diamond as a thermoluminescent dosemeter, Diamond Relat. Mater. 9, 1013-1016.

Benabdesselam M., Iacconi P. (2001) Effets de l'excitation optique UV-visible sur la thermoluminescence des films de diamant CVD. Dans : $18^{e s}$ Journées des LARD, 18-19 octobre 2001, Barcelone (Espagne).

Benabdesselam M., lacconi P., Butler J.E., Nigoul J.M. (2003) TL characterization of a CVD diamond wafer for ionising radiation dosimetry, Diamond Relat. Mater. 12, 1750-1754.

Benabdesselam M., Serrano B., Iacconi P., Wrobel F., Lapraz D., Herault J., Butler J.E. (2004) Thermoluminescence properties of CVD diamond for clinical dosimetry use. In: 14th International Conference on Solid State Dosimetry, 2004, New Haven (USA).

Berkane-Krachai K. (2001) Modélisation et simulation de phénomènes prenant en compte le gradient thermique dans l'échantillon et l'extinction thermique de la luminescence. Application à l'alumine alpha, Thèse de l'université de Nice-Sophia Antipolis.

Berkane-Krachai A., Iacconi P., Bindi R., Vinceller S. (2002) Analysis with a multilayer model of heating rate effect on thermally stimulated luminescence, conductivity and exoelectronic emission of $\alpha-\mathrm{Al}_{2} \mathrm{O}_{3}$, J. Phys. D: Appl. Phys. 35, 1895-1902.

Bindi R., Lapraz D., Boutayeb S., Iacconi P. (1994) Theoretical analysis of the simultaneous detection method of thermally stimulated conductivity (TSC) and luminescence (TSL); application to an $\alpha-\mathrm{Al}_{2} \mathrm{O}_{3}$ monocrystal, J. Phys. D: Appl. Phys. 27, 2395-2400.

Bindi R., Iacconi P., Lapraz D., Petel F. (1997) Effective electron estimation from the simultaneous detection of thermally stimulated luminescence and exoelectronic emission. Application to an $\alpha-\mathrm{Al}_{2} \mathrm{O}_{3}$ sample, J. Phys. D: Appl. Phys. 30, 137-143.

Bourhis E. (2000) Appplication de codes Monte Carlo à la dosimétrie de la potentialisation par capture de neutrons par le bore 10. Mise en œuvre en radiothérapie du cancer, Thèse de l'université de Nice-Sophia Antipolis.

Bourhis-Martin E., Hachem H., Herault J., Paul D., Paquis P. (2002) Application of Monte Carlo calculation codes to the dosimetry of boron neutron. In: 10th Intern. Congress on Neutron Capture Therapy, 8-13 september 2002 Essen (Allemagne).

Boutayeb S. (1993) Conductibilité électrique thermostimulée de l'alumine alpha entre 77 et $900 \mathrm{~K}$. Étude et caractérisation d'un phénomène photoinduit, Thèse de l'université Paul SabatierToulouse.

Ferey F., Grosseau P., Guilhot B., Iacconi P., Benabdesselam M. (2001) Thermoluminescence and sintering of high-purity $\alpha$-alumina doped by Zr, Th and Ca, Solid State Ionics 141/142, 567-574.

Ferey F. (2002) Thermoluminescence et frittage de l'alumine alpha ultra pure dopée par le zirconium, le thorium, le calcium ou le cérium, Thèse des l'École des Mines de St Étienne. 
Ferey F., Grosseau P., Guilhot B., Iacconi P., Benabdesselam M. (2002) Thermoluminescent and sintering's behaviour of ultra-pure alpha alumina doped by $\mathrm{Th}, \mathrm{Zr}$, Ce or Ca. Euro Ceramics VII (C. Kernel, V. Lardot, D. Libert, I. Urbain, Eds) Key Engineering Materials, 206/213, 115-118.

Franchisseur E., Serrano B., Hachem H., Bensadoun R., Monnier A., Barthe J. (2004) Applications des codes MonteCarlo «MNCP » et «PENELOPE » en radiothérapie du cancer. Dans : $21^{e s}$ Journées des LARD, 21-22 octobre 2004, Limoges.

Gerôme V., Iacconi P., Lapraz D., Prevost H., Baumer A. (1996) Thermoluminescence of undoped $\mathrm{CaSO}_{4}$ and $\mathrm{CaSO}_{4}$ :Dy. Influence of the preparation methods, Radiat. Prot. Dosim. 65, 309-312.

Gerôme V. (1997) Étude et interprétation des mécanismes thermostimulés présentés par le sulfate de calcium dopé par des éléments trivalents de terres rares, Thèse de l'université Paul SabatierToulouse.

Goyet D. (1993) Étude des propriétés thermoluminescentes du $\mathrm{CaSO}_{4}$ :Dy. Dosimétrie des rayonnements ionisants à haute température, Thèse de l'université Paul Sabatier-Toulouse.

Goyet D., Lapraz D., Iacconi P., Portal., Barthe J., Prevost H., Gasiot J. (1993) UV Emission in the TL peaks of $\mathrm{CASO}_{4}$ :Dy. Origin and Application to high Temperature Dosimetry, Radiat. Prot. Dosim. 47, 147-150.

Goyet D., Lapraz D., Iacconi P. (1996) Application of $\mathrm{CaSO}_{4}$ :Dy PTTL phenomenon to high temperature dosimetry, Radiat. Prot. Dosim. 4, 317-320.

Guissi S. (1998) Modélisation des phénomènes thermostimulés. Validation par la détection simultanée de la luminescence, de la conductivité électrique et de l'émission exoélectronique thermostimulées dans l'alumine alpha, Thèse de l'université de Nice-Sophia Antipolis.

Guissi S., Bindi R., Iacconi P., Jeanbrun D., Lapraz D. (1998) Theoretical model for thermally stimulated luminescence, conductivity and exoelectronic emission, J. Phys. D: Appl. Phys. 31, 136-144.

Guissi S., Iacconi P., Bindi R., Lapraz D. (1999) Characterisation of the 450 K TSL peak of alpha alumina by the simultaneous analysis of TSL, TSC and TSEE, Radiat. Prot. Dosim. 84, 247251.

Iacconi P., Lapraz D., Keller P., Portal G., Barthe J. (1986) Photoinduced thermoluminescence of Xirradiated alpha- $\mathrm{Al}_{2} \mathrm{O}_{3}$ : Dosimetric properties, Radiat. Prot. Dosim. 17, 475-478.

Iacconi P. Lapraz D., Alessandri-Fraccaro M.F., Addi D. (1990) Phototransferred Thermoluminescence and Exoemission in alpha-alumina, Radiat. Prot. Dosim. 33, 127-130.

Iacconi P., Petel F., Lapraz D., Bindi R. (1993) Thermostimulated Exoelectronic Emission and Thermoluminescence of various $\alpha-\mathrm{Al}_{2} \mathrm{O}_{3}$ samples, Phys. Stat. Sol. (a) 139, 497-501.

Iacconi P., Lapraz D., Bindi R., Benabdesselam M. (1997) Photoinduced effect in $\alpha$-alumina. Characterisation by TSEE method, Phys. Stat. Sol. (b) 163, 337-348.

Lapraz D., Iacconi P. (1985) Photo Induced Thermoluminescence of X-Irradiated alpha- $\mathrm{Al}_{2} \mathrm{O}_{3}$, Phys Stat. Sol. (a) 108, 783-794.

Lapraz D., Iacconi P., Sayadi Y., Keller P., Barthe J., Portal G. (1988) Some thermoluminescence properties of an alpha- $\mathrm{Al}_{2} \mathrm{O}_{3}$. Sample sensitization effects, Phys. Stat. Sol. (a) 87, K167-169.

Lapraz D., Iacconi P., Alessandri-Fraccaro M.F., Barthe J., Portal G. (1990) Dosimetric Characteristics of Specially Prepared Clothing Material. In: 9th. Int. Conf. Solid State Dosimetry, Nov. 1989, Vienne (Autriche), Radiat. Prot. Dosim. 33, 131-134.

Lapraz D., Iacconi P., Daviller D., Guilhot B. (1991) Thermostimulated Luminescence and Fluorescence of $\alpha-\mathrm{Al}_{2} \mathrm{O}_{3}: \mathrm{Cr}^{3+}$ (ruby), Phys. Stat. Sol. (a) 26, 521-531.

Lapraz D., Boutayeb S., Iacconi P., Bindi R., Rostaing P. (1993) Photoinduced Thermostimulated Electrical Conductivity of an $\alpha-\mathrm{Al}_{2} \mathrm{O}_{3}$ monocrystals, Phys. Stat. Sol. (a) 136, 497-507.

Lapraz D., Goyet D., Iacconi P., Gerôme V., Prevost H., Gasiot J., Baumer A. (1996) UV emissions in the thermostimulated luminescence of $\mathrm{CaSO}_{4}: \mathrm{Dy}^{3+}$; influence of the host $\mathrm{CaSO}_{4}$ lattice, $\mathrm{Dy}^{3+}$ concentration and $\mathrm{Tm}^{3+}$ codoping, Phys. Stat. Sol. (a) 153, 239-247. 
Lapraz D., Prevost H., Baumer A., Iacconi P., Benabdesselam M., Blanc P. (2000) Effect of Thermal Preannealing on Fluorescence, Thermostimulated Luminescence and Cathodoluminescence of $\mathrm{CaSO}_{4}$ Doped with Europium, Phys. Stat. Sol. (a) 181, 515-527.

Lapraz D., Prevost H., Iacconi P., Guigues C., Benabdesselam M., Briand D. (2002) On the Luminescence Properties of $\mathrm{CaSO}_{4}$ :Ce, Radiat. Prot. Dosim. 100, 365-368.

Lapraz D., Prevost H., Lakshmanan A.R. (2004) Instability of $\mathrm{Eu}^{2+} / \mathrm{Eu}^{3+}$ photoluminescence in Xirradiated $\mathrm{CaSO}_{4}: \mathrm{Eu}^{3+}$, Phys. Stat. Sol. (a) 201, 596-601.

Lakshmanan A.R., Lapraz D., Prevost H., Benabdesselam M. (2005) Thermostimulated Luminescence Properties of $\mathrm{CaSO}_{4}$ :Dy and $\mathrm{CaSO}_{4}: \mathrm{Tm}$ Phosphors Annealed at High Temperatures, Phys. Stat. Sol. (a) 202, 131-139.

Mady F., Iacconi P., Bindi R. (2005) Modélisation de la TL et interprétation expérimentale. Utilité d'une analyse globale de la réponse en fonction de la dose, Dans : $22^{\text {es }}$ journées des LARD, 2021 octobre 2005, Montbéliard.

Mady F., Bindi R., Iacconi P., Wrobel F. (2006) Influence of the Absorbed Dose and of Deep Traps on Thermally Stimulated Phenomena : a Numerical Simulation, Radiat. Prot. Dosim. 119, 37-40.

Marcié S., MartinE., Bensadoun R., Magné N., Herault J., Costa A., Hachem H., Lagrange J.L. (2003) Study of theoretical cases of intensity modulated beams with step-and-shoot technique, CancerRadiothérapie 7, 179-183.

Martin E. (2002) Validation par mesures physiques et modélisation de la radiothérapie conformationnelle avec modulation d'intensité sur les systèmes dosimétriques Helax TMS et l'accélérateur Primus, Thèse de l'université de Nice.

Martin E., Hachem H., Marcié S., Herault J., Costa A., Bensadoun R., Lagrange J.L. (2003) Validity of intensity modulation on a commercial treatment planning system, Medical Phys. 30, 925-936.

Molnar G., Papin E., Grosseau P., Guilhot B., Borossay J., Benabdesselam M., Iacconi P., Lapraz D. (1999) Thermally Stimulated Luminescence and Exoelectron Emission Mechanism of the 430 K Dosimetric Peak of $\alpha-\mathrm{Al}_{2} \mathrm{O}_{3}$, Radiat. Prot. Dosim. 84, 253-256.

Molnar G. (2000) Étude du rôle des défauts ponctuels dans la thermoluminescence de monocristaux d'alumine alpha. Effets des traitements thermiques, Thèse de l'université de Nice-Sophia Antipolis.

Molnar G., Borossay J., Benabdesselam M., Iacconi P., Lapraz D., Süvegh K., Vertes A. (2000) Oxydation/reduction effects on the TL of $\alpha-\mathrm{Al}_{2} \mathrm{O}_{3}$ single crystals, Phys. Stat. Sol. (a) 179, 249-260.

Molnar G., Benabdesselam M., Borossay J., Iacconi P., Lapraz D., Akselrod M. (2002) Influence of the irradiation temperature on the dosimetric and high temperature TL peaks of $\mathrm{Al}_{2} \mathrm{O}_{3}: \mathrm{C}$, Radiat. Prot. Dosim. 100, 139-142.

Papin E., Grosseau P., Guilhot B., Benabdesselam M., Lapraz D., Iacconi P. (1996) Influence of the calcining conditions on the thermoluminescence of pure and doped $\alpha$-alumina powders, Radiat. Prot. Dosim. 65, 243-246.

Papin E. (1997) Influence des défauts ponctuels sur les propriétés dosimétriques et sur l'aptitude au frittage de l'alumine alpha, Thèse de l'École des Mines de St. Étienne.

Papin E., Grosseau P., Guilhot B., Benabdesselam M., Iacconi P. (1997) Point defects in alumina studied by thermoluminescence, Proc. of Eurosolid 4 (A. Negro, L. Montanaro, Eds) pp. 158163, ISBN 88-8202-003-7.

Papin E., Grosseau P., Guilhot B., Benabdesselam M., Iacconi P. (1999) Influence of point defects on the thermoluminescence of $\alpha-\mathrm{Al}_{2} \mathrm{O}_{3}$ : Application to dosimetry, Radiat. Prot. Dosim. 84, 91-94.

Petel F. (1994) Détection simultanée de l'émission exoélectronique et de la luminescence thermostimulées présentées par l'alumine alpha entre 77 et $650 \mathrm{~K}$, Thèse de l'université Paul Sabatier-Toulouse. 
Petel F., Iacconi P., Bindi R., Lapraz D., Breuil P. (1996) Simultaneous detection of thermostimulated luminescence and exoelectronic emission between 77 and $650 \mathrm{~K}$. Application to alpha alumina, Radiat. Prot. Dosim. 65, 247-250.

Petitfils A., Wrobel F., Benabdesselam P., Iacconi P., Serrano B., Costa A. (2005) Application du diamant CVD en mode TL pour la radiothérapie. Propriétés de thermoluminescences et de conductivité électrique. Dans : $22^{\text {es }}$ Journées des LARD, 20-21 octobre 2005, Montbéliard.

Salle C. (2003) Effets du Zr, du Mn et du Ti sur la thermoluminescence et la réactivité de l'alumine alpha, Thèse de l'École des Mines de St. Étienne.

Sayadi Y. (1988) Étude et caractérisation d'un phénomène de TL photoinduite dans l'alumine alpha. Application à la dosimétrie des rayonnements ionisants, Thèse de l'université Paul SabatierToulouse.

Serrano B. (2006) Application du code Monte Carlo à la radiothérapie conformationnelle avec modulation d'intensité sur le système dosimétrique Helax TMS. Application à la sphère ORL, Thèse de l'université de Nice-Sophia Antipolis.

Vinceller S. (2002) Effets de l'extinction thermique de la luminescence sur les phénomènes thermostimulés présentés par le pic dosimétrique de l'alumine alpha, Thèse de l'université Paul Sabatier-Toulouse.

Vinceller S., Molnar G., Berkane-Krachai A., Iacconi P. (2002) Influence of thermal quenching of the thermostimulated processes in $\alpha-\mathrm{Al}_{2} \mathrm{O}_{3}$. Role of $\mathrm{F}$ and $\mathrm{F}+$ centres, Radiat. Prot. Dosim. 100, 79-82.

Wrobel F., Palau J.-M., Calvet M.C., Iacconi P. (2003) Contribution of $\mathrm{SiO}_{2}$ in neutron induced SEU in SRAMS, IEEE-Trans. Nucl. Science 50, 2055-2059.

Wrobel F., Palau J.-M., Iacconi P., Palau M.-C., Sagnes B., Saigné F. (2004a) Methodologie to compute neutron induced alphas contribution on the SEU cross section in sensitive SRAMS, IEEE-Trans. Nucl. Science 51, 3225-3230.

Wrobel F., Benabdesselam M., Iacconi P., Lapraz D. (2004b) Neutron Dose Evaluation by Calculations of Recoil Energy. In: 9th Symp. Intern. neutron Dosimetry, 28 sept.-3 oct. 2003, Delft (Pays Bas), Radiat. Prot. Dosim. 110, 807-811.

Wrobel F., Iacconi P. (2005) Parametrization of neutron induced SER in bulk SRAMS from reverse Monte Carlo simulations. In: NSREC 2005, 11-15 juillet 2005, Seattle, Washington (USA), IEEE-Trans. Nucl. Science 52, 2313-2318. 